\title{
Intraoperative Rupture of Aneurysm: Does It Add Insult to the Injury?
}

\author{
Ganne S. Umamaheswara Rao ${ }^{1}$ \\ ${ }^{1}$ Department of Neuroanaesthesia and Critical Care, National \\ Institute of Mental Health and Neurosciences (NIMHANS), \\ Bengaluru, Karnataka, India
}

J Neurosci Rural Pract 2021;12:224-225.

Subarachnoid hemorrhage due to rupture of cerebral aneurysm is by itself a devastating condition. Unexpected rupture of an intracranial aneurysm during surgery is considered a potentially dangerous event. There is wide-ranging and conflicting literature on this issue.

The reported incidence of intraoperative rupture in the literature varies significantly. After the advent of operating microscope, the reported incidence of intraoperative aneurysm rupture (IAR) is around $20 \% .{ }^{1}$ Another Japanese study which analyzed the outcome of 398 ruptured aneurysms reported an incidence of $6.0 \% .^{2}$ In another series of 1,694 aneurysms, the incidence was $4.3 \%$ per patient. The rate was greater in ruptured than unruptured aneurysms (10.7 vs. $1.2 \%)^{3}$ Patients subjected to surgery within 72 hours and after 72 hours of IAR were 40.2 and $20.7 \%$, respectively. ${ }^{4}$ The location of aneurysm and rupture status of the aneurysm also seem to influence IAR. In one series, the highest rate of IAR was in patients with internal carotid artery (ICA) aneurysms (25\%). ${ }^{5}$

Rupture generally occurs at the following three stages during surgery: (1) predissection, (2) during dissection, and (3) during clipping.

Different studies attempted to find out the predictive factors for IAR. In a series of 182 patients with a ruptured middle cerebral artery (MCA) aneurysm, 11 patients (6.0\%) suffered IAR. A binary logistic regression revealed that presence of a sphenoid ridge proximation sign (defined as a spatial proximation of $<4 \mathrm{~mm}$ between the sphenoid ridge and the rupture point of the MCA aneurysm; $p<0.001$ ), presence of a frontal intracerebral hematoma (ICH; $p=0.019$ ), and a short preaneurysmal $\mathrm{M}_{1}$ segment $(p=0.043)$ were statistically significant risk factors for premature rupture. ${ }^{6}$

In a series of 903 patients, a multivariate analysis revealed ruptured status (adjusted odds ratio [OR] of 10.46; $p<0.001$ ), sack size (adjusted $\mathrm{OR}=1.05$ per $\mathrm{mm}$ increase; $p=0.038$ ), and aneurysm location in the anterior communicating artery

Address for correspondence Ganne S. Umamaheswara Rao, MD, Department of Neuroanaesthesia and Critical Care, National Institute of Mental Health and Neurosciences (NIMHANS), Bengaluru 560029, Karnataka, India (e-mail: gsuma123@yahoo.com). (adjusted $\mathrm{OR}=2.31 ; p<0.001)$ independently predicted IAR. Rebleeding before therapy (adjusted $\mathrm{OR}=3.11 ; p=0.033$ ) and clinical severity of subarachnoid hemorrhage (adjusted OR = 1.18 per World Federation of Neurological Surgeons grade increase; $p=0.049$ ) also predicted IAR. ${ }^{7}$

Premature rupture can be managed by the following: (1) temporary arterial occlusion, (2) tamponade using cotton and suction, (3) suction dissection of the aneurysm, and (4) clip application to the distal sac or coagulation of the aneurysmal rent.

Elective temporary clipping (ETC) is used in surgery for aneurysms to prevent this complication which has decreased the occurrence of IAR leading to significantly better outcome. Independent of other factors, ETC less than 20 minutes resulted in significantly better outcome. ${ }^{8}$ In a retrospective study of temporary clipping of 338 (43.4\%) out of 778 patients, temporary clipping did not contribute to a higher rate of transcranial Doppler (TCD) proven vasospasm, delayed ischemic neurologic deficit (DIND), or delayed cerebral ischemia (DCI). In contrast, the need for rescue temporary clipping was a predictor for DCI (19.5\% in the rescue clipping group vs. $11.3 \%$ in the elective clipping group; $p=0.02$ ). ${ }^{9}$

Some studies attempted to identify the anatomical features of the aneurysm prone for intraoperative rupture. In one study, aneurysms were divided into translucent aneurysms (TA) defined as $>90 \%$ reddish pigmentation appearance of the aneurysm wall or non-TA (NTA) according to the observation under microscope. TAs were associated with intraoperative rupture (28.6 vs. $0 \%, p=0.04){ }^{10}$

Patient-specific three-dimensional (3D) aneurysm models were constructed from preoperative computed tomography angiography (CTA) data, and computational fluid dynamics (CFD) analyses were performed under pulsatile-flow conditions. Hemodynamic parameters derived from CFD analysis, including normalized wall shear stress (NWSS), normalized pressure (NP), oscillatory shear index (OSI), and relative residence time (RRT),

(c) 2021. Association for Helping Neurosurgical Sick People.

This is an open access article published by Thieme under the terms of the Creative Commons Attribution-NonDerivative-NonCommercial-License, permitting copying and reproduction so long as the original work is given appropriate credit. Contents may not be used for commercial purposes, or adapted, remixed, transformed or built upon. (https://creativecommons.org/licenses/by-nc-nd/4.0/).

Thieme Medical and Scientific Publishers Pvt. Ltd. A-12, 2nd Floor, Sector 2, Noida-201301 UP, India 
were compared between thin-walled regions and surrounding normal-thickness areas. Thin-walled regions of aneurysm wall tended to present with higher pressure (1.232 vs. 1.043 , $p<0.05$ ) and lower wall shear stress (0.693 vs. $0.868, p<0.05$ ). Higher pressure and lower WSS were characteristic hemodynamic features associated with thinner regions of the aneurysm wall. Elevated NP was an independent risk factor for local aneurysm wall thinning. Thus, CFD seems to be a useful method to estimate the location of thin-walled region. ${ }^{11}$

Induced hypotension was routinely used before clipping of the aneurysm earlier. A few series have shown poor outcome with this approach. ${ }^{12}$ In 41 intraoperative ruptures during 276 surgeries, the authors concluded that hypotension may not be a necessary in the management of intraoperative rupture of aneurysms. ${ }^{13}$

The crux of the issue regarding IAR is whether IAR enhances the risk of unfavorable outcome of the surgery. There is variable opinion. During surgery on anterior cerebral artery (ACA) or anterior communicating artery $(\mathrm{ACOA})$ aneurysms, IAR affected final outcome only when it occurred prematurely during induction of anesthesia or during opening of the dura. ${ }^{4}$ In another series, poor initial clinical conditions (Hunt and Hess grades IV and V), as well as the initial Fisher's grades III and IV, were strongly associated with poor clinical outcome..$^{14}$ In a PubMed search of more than 10 years, intraoperative rupture during predissection phase is associated with poor outcome..$^{15}$

A few pharmacological attempts have been made recently to decrease the incidence of IAR or to decrease the adverse side effects of IAR. In a small series of 18 patients, recombinant factor VII was used to prevent intraoperative rupture. None of the patients had IAR. The drug was well tolerated. ${ }^{16}$ In a series of aneurysm patients, adenosine-induced circulatory arrest was shown to be a safe option in 16 patients who had IAR. But a very close communication between the surgeon and the anesthesiologist is required to produce a controlled cardiac arrest. ${ }^{17}$

In the current issue of Journal of Neurosciences in Rural Practice, the authors studied the effects of intraoperative aneurysm rupture on perioperative complications and neurological outcome. ${ }^{18}$ The authors could not find any preoperative predictive factors for IAR. But they found that the neurological outcome was worse in patients with IAR. Overall the outcome variables were more or less similar to what have been reported in the literature. On multivariate analysis, the authors also found that the usage of colloids was significantly more and the duration of surgery was prolonged in patients with IAR which can be expected. An in-depth analysis of the factors/reasons for poor outcome in the IAR group would have contributed more to the literature. But the limitations of a retrospective study could well be understood.

\section{Conflict of Interest}

None declared.

\section{References}

1 Chandler JP, Getch CC, Batjer HH. Intraoperative aneurysm rupture and complication avoidance. Neurosurg Clin N Am 1998;9(4):861-868
2 Houkin $\mathrm{K}$, Kuroda S, Takahashi A, et al. Intra-operative premature rupture of the cerebral aneurysms. Analysis of the causes and management. Acta Neurochir (Wien) 1999;141(12):1255-1263

3 Leipzig TJ, Morgan J, Horner TG, Payner T, Redelman K, Johnson CS. Analysis of intraoperative rupture in the surgical treatment of 1694 saccular aneurysms. Neurosurgery 2005;56(3):455-468, discussion 455-468

4 Schramm J, Cedzich C. Outcome and management of intraoperative aneurysm rupture. Surg Neurol 1993;40(1):26-30

5 Chen SF, Kato Y, Kumar A, et al. Intraoperative rupture in the surgical treatment of patients with intracranial aneurysms. J Clin Neurosci 2016;34:63-69

6 Park J, Son W, Park KS, Kang DH, Shin IH. Intraoperative premature rupture of middle cerebral artery aneurysms: risk factors and sphenoid ridge proximation sign. J Neurosurg 2016;125(5):1235-1241

7 Darkwah Oppong M, Pierscianek D, Ahmadipour Y, et al. Intraoperative aneurysm rupture during microsurgical clipping: risk re-evaluation in the post-international subarachnoid aneurysm trial era. World Neurosurg 2018;119:e349-e356

8 Dhandapani S, Pal SS, Gupta SK, Mohindra S, Chhabra R, Malhotra SK. Does the impact of elective temporary clipping on intraoperative rupture really influence neurological outcome after surgery for ruptured anterior circulation aneurysms?-A prospective multivariate study. Acta Neurochir (Wien) 2013;155(2):237-246

9 Malinova V, Schatlo B, Voit M, Suntheim P, Rohde V, Mielke D. The impact of temporary clipping during aneurysm surgery on the incidence of delayed cerebral ischemia after aneurysmal subarachnoid hemorrhage. J Neurosurg 2018;129(1):84-90

10 Chen XL, Chen Y, Ma L, et al. Translucent appearance of middle cerebral artery bifurcation aneurysms is risk factor for intraoperative aneurysm rupture during clipping. World Neurosurg 2017;101:149-154

11 Jiang P, Liu Q, Wu J, et al. Hemodynamic characteristics associated with thinner regions of intracranial aneurysm wall. J Clin Neurosci 2019;67:185-190

12 Drummond JC. Deliberate hypotension for intracranial aneurysm surgery: changing practices. Can J Anaesth 1991;38(7):935-936

13 Giannotta SL, Oppenheimer JH, Levy ML, Zelman V. Management of intraoperative rupture of aneurysm without hypotension. Neurosurgery 1991;28(4):531-535, discussion 535-536

14 Sandalcioglu IE, Schoch B, Regel JP, et al. Does intraoperative aneurysm rupture influence outcome? Analysis of 169 patients. Clin Neurol Neurosurg 2004;106(2):88-92

15 Chowdhury T, Cappellani RB, Sandu N, Schaller B, Daya J. Perioperative variables contributing to the rupture of intracranial aneurysm: an update. ScientificWorldJournal 2013;2013:396404

16 Nussbaum ES, Janjua TM, Defillo A, Sinner P, Zelensky A. Perioperative use of recombinant factor VII to prevent intraoperative aneurysm rupture in high risk patients: a preliminary safety evaluation. Neurocrit Care 2009;10(1):55-60

17 Luostarinen T, Takala RS, Niemi TT, et al. Adenosine-induced cardiac arrest during intraoperative cerebral aneurysm rupture. World Neurosurg 2010;73(2):79-83, discussion e9

18 Radhakrishna N, Khandelwal A, Chouhan RS, et al. Complications and neurological outcome following intraoperative aneurysm rupture in adult patients undergoing intracranial aneurysmal clipping: a retrospective study. J Neurosci Rural Pract 2021;12(2):382-388 